\title{
Probabilistic Priority Assessment of Nurse Calls
}

Femke Ongenae ${ }^{1, *}$, Msc., Dries Myny ${ }^{2, * *}$, PhD RN, Tom Dhaene ${ }^{1}$, Prof. PhD, Tom Defloor ${ }^{3}$, Prof. PhD RN, Dirk Van Goubergen ${ }^{4}$, Prof. PhD, Piet Verhoeve ${ }^{5}, \mathrm{PhD}$, Johan Decruyenaere ${ }^{6}$, Prof. PhD MD, Filip De Turck ${ }^{1}$, Prof. PhD

${ }^{1}$ Ghent University - IBBT, Department of Information Technology (INTEC), Gaston Crommenlaan 8, bus 201, 9050 Ghent, Belgium

${ }^{2}$ Ghent University Hospital, Nursing department, De Pintelaan 185, 9000 Ghent, Belgium

${ }^{3}$ Ghent University, Faculty of Medicine and Health Sciences, Nursing Science, Ghent, Belgium, Pintelaan 185, 9000 Ghent, Belgium

${ }^{4}$ Ghent University, Department of Industrial Management, Technology Park 903, 9052 Ghent, Belgium

${ }^{5}$ Televic R\&D, Leo Bekaertlaan 1, 8870 Izegem, Belgium

${ }^{6}$ Ghent University Hospital, Intensive Care Department, De Pintelaan 185, 9000 Ghent, Belgium

Femke Ongenae is funded by the Institute for the Promotion of Innovation by Science and Technology in Flanders (IWT).

\section{Word count:}

- Abstract: 246 words

- Main text: 7,929 words

\footnotetext{
* Corresponding author. Tel.: +32 933 14938; fax: +32 933 14899. E-mail address: Femke.Ongenae@ @intec.ugent.be

** Corresponding author. Tel.: +32 933 26412; fax: +32 933 23325. E-mail address: Dries.Myny@uzgent.be
} 


\section{Abstract}

The current nurse call systems are very static. The call buttons are fixed to the wall. Additionally, these systems do not take into account various factors specific to a situation. We have developed a software platform, the oNCS, which supports the transition to mobile and wireless nurse call buttons and employs an intelligent nurse call algorithm. This algorithm dynamically adapts to the situation at hand by taking the profile information of staff and patients into account by employing an ontology. This paper describes a probabilistic extension of this oNCS that supports a more sophisticated nurse call algorithm by dynamically assigning priorities to calls based on the risk factors of the patient and the kind of call.

The probabilistic oNCS is evaluated through a prototype implementation and simulations, based on a detailed dataset obtained from 3 nursing departments of Ghent University Hospital. The arrival times of nurses at the location of a call, the workload distribution of calls amongst nurses and the assignment of priorities to calls are compared for the oNCS and the current nurse call system. Additionally, the performance of the system and the parameters of the priority assignment algorithm are explored.

The execution time of the nurse call algorithm is on average $50.333 \mathrm{~ms}$. Moreover, the probabilistic oNCS significantly improves the assignment of nurses to calls. Calls generally have a nurse present faster, the workload-distribution amongst the nurses improves and the priorities and kinds of calls are taken into account.

\section{Introduction}

In recent years the complexity of continuous care has been increasing due to the increase of the care unit size, specialized care and combined care paths. The lack of nurse staffing also requires a more efficient use of resources. To deal with these issues, information technology (IT) is often used. IT has already proven its merit in other healthcare fields [1][2][3].

Observations and contextual inquiries at a representative hospital setting at the start of this research [4][5] revealed that ICT could greatly contribute to continuous care by performing 1) information integration \& data provisioning at the point of care and 2) by supporting communication between both 
the staff and the staff and patients. The first can be summarized as providing the right information, at the right time, at the right place for the right person. This requires an increased need for mobile services [6] to support data input, e.g., care registration, and request data, which should be integrated, prioritized and filtered based on contextual information.

Regarding the second, it was found that a nurse call system is an important way to coordinate work, communicate and provide continuous care. When patients feel unwell they push a button. The nurses receive a message on a beeper with the room number. This brings the question: which nurse goes to the room, the closest one, the one on call, etc.? The caregivers use the nurse call system to be alerted of patient's needs, communicate with them through intercoms and request help from other staff.

Traditional nurse call systems are static as calls are made by buttons fixed to a wall and the assignment algorithm consists of predefined links between rooms and caregivers' beepers. Herewith two assumptions are made: the patient must still be in the room when the assigned nurse arrives and it must be the patient who lies in the room that made the call. The current systems thus do not take into account the specific situation and context, e.g., risk factors of a patient or locations of the staff, to assign nurses to calls. The beepers give the nurses limited context information about the call. They need to go to the room to determine the urgency and reason of the call and whether they need additional care products to handle it. This leads to extra miles for the caregivers and a need to interrupt their current tasks to assess the call. Nurses are also not aware of each other's tasks and whether a caregiver already has the intention to handle the call. This causes that multiple nurses arrive at a room to handle a call and thus that their tasks were unnecessarily interrupted. Moreover, it is dangerous for patients to become unwell inside a hallway, staircase or outside as they cannot call someone in these areas. This leads to patients being confined to their room to ensure their safety.

A trend is emerging towards nurse call systems equipped with a mobile button for each patient so that they can walk around freely [7][8]. Novel systems also equip each room with a terminal screen. However, this screen is currently not used to display relevant context data.

It is clear that continuous care could greatly benefit from the incorporation of a context-aware nurse call system, which uses integrated context information about the staff and patients, e.g., locations and qualifications, to assign the appropriate nurse to the patient. This way the communication and 
workflows could be dynamically optimized. This is necessary as calls are unforeseen tasks with a wide variety of reasons and priority, which makes them difficult to assign and schedule in advance. Moreover, the relevant context information pertaining to the call could be visualized to the assigned caregiver and data input about the call could be supported at the point of care.

To realize this vision, we have designed the ontology-based Nurse Call System (oNCS). This platform allows that patients walk around freely with portable, wireless call buttons. Additionally, this platform efficiently manages the profiles of staff members and patients, e.g., roles, competences, risk factors, current location and tasks, by encoding this information into an ontology [9]. An ontology formally models the concepts and their relationships and properties within a domain. A new nurse call algorithm was developed, which dynamically adapts to the situation at hand by taking this profile information into account, to find the best caregiver to handle a specific call. This makes the system more adaptable to the needs and preferences of patients and staff. A description of this platform can be found in Ongenae, et al. [10].

The goal of this paper is two-fold. First, an extension of the oNCS is described in Section 2, which supports a more sophisticated nurse call algorithm by dynamically assigning priorities to calls based on the risk factors of patients and the type of call. As patients with a particular profile can still make calls of varying priority, this information is modeled probabilistically in the ontology. By using probabilistic reasoning and threshold algorithms, these probabilistic values are combined to determine the most suitable priority for a call. These priorities are taken into account when a suitable nurse is searched to handle a call. Second, an extensive simulation with realistic data about 3 departments of Ghent University Hospital [11] is presented in Section 3. It demonstrates the advantages and performance of the system and explores the sensitivity of the threshold algorithm. A critical discussion of the platform and its expected benefits is presented in Section 4. Finally, Section 5 highlights the conclusions. This paper focuses on the probabilistic modeling of the data and accompanying probabilistic reasoning algorithms. An overview of the design of the complete system can be found in Ongenae, et al. [12]. 


\section{Methods}

\subsection{Profile management}

In order to achieve a nurse call algorithm that adapts to the situation at hand, an ontology is used to efficiently manage the context information. From field trials and experiments it was derived which context information is relevant. The most important concepts and relations of this ontology are visualized in Figure 1. First, the different types of staff members, e.g., nurse or caretaker, and their properties, e.g., location, status (free or busy) and current task, were modeled. Second, information about the patients was modeled, e.g., location and risk factors. Finally, the various types of calls and tasks were modeled. Three kinds of calls can be launched by patients. Normal calls are made for medical problems and service call for a "caring" tasks, e.g., asking for water. Sanitary calls originate from a sanitary space. Urgency, medical, technical and (sanitary) assistance calls are launched by nurses. For each call it is also indicated which types of staff may handle. Each task and call has an associated priority. A detailed description of this ontology can be found in Ongenae, et al. [12].

This ontology was extended with profile information to probabilistically determine the priority of a call. First, based on input from medical and nurse call experts, risk factors were added to the ontology as shown at the top of Figure 1, e.g., heart patient, diabetic or elderly. They have an impact on the priority of calls made by patients that exhibit these risks. Second, to express this impact, probabilistic information is added to the ontology. This information expresses the probability that a particular patient belongs to a particular risk group, namely high, medium and low risk patients, based on his risk factors and the department he resides on. For example, a patient with a neurological problem has at least $50 \%$ and at most $30 \%$ and $20 \%$ chance of being a high, medium and low risk patient respectively. Third, the probabilistic assignment of patients to risk groups is used to determine the priority of calls made by or for these patients. There are seven priorities: highest, high, above normal, normal, below normal, low and lowest. The call's priority is also based on its type, e.g., normal or sanitary. So when a patient from a particular risk group, makes a particular type of call, this call is assigned a probability of having a specific priority. For example, when a high risk patient makes a normal call, this call has respectively $20 \%, 60 \%$ and $20 \%$ chance of being of high, above normal and normal priority. It has $0 \%$ chance of receiving the other priorities. 
The priority of urgency, medical and technical calls does not depend on the profile of patients. They get the highest, low and lowest priority respectively.

\subsection{Algorithms}

As illustrated in Figure 2, algorithms were constructed to assign the best possible nurse to a call. First, the priority of the call is determined by reasoning on the probabilistic information in the ontology. The probabilistic priority assessment algorithm, detailed in Section 2.2.1, determines for each priority category the probability that this call has this priority. A threshold algorithm is then used to associate the most probable priority with the call, based on these probabilities. Finally, as discussed in Section 2.2.2, the priority of the call is combined with the other context information captured in the ontology to assign the most suitable nurse to the call.

\subsubsection{Priority assessment of a call}

To assess the priority of the call based on the information in the ontology, probabilistic reasoning is first used to calculate for each of the seven possible priorities, the probability that this call has this priority. To determine the suitable priority for the call based on these probabilities, a threshold algorithm is used. If the probabilistic value for the priority is higher than or equal to the threshold for this priority, it gets this priority.

The thresholds for each priority for a particular hospital or department are determined by running computer simulations based on data gathered about this hospital or department. First, the risk profiles of the patients within this department are determined and weights are assigned to these risk factors that reflect how frequently they occur. Combinations of risk factors that are deemed to be more frequent than others are also specified. For example, the risk factors neurological problem and disoriented/confused often occur together, e.g., in patients with multiple sclerosis. In some departments certain combinations may be encountered more frequently than in others. The risk profiles used in this research are discussed in Section 2.3.

Second, 20 test and 10 validation groups of patients with risk factors are randomly generated, using the algorithm visualized in Figure 3. Each group contains as many patients as there are beds within the department. Flow B takes the possible combinations into account. If a risk factor is chosen, then all the 
weights of the risk factors that it can have a combination with are doubled. It is possible that a weight of a risk factor is doubled twice if it both occurs in the general and department specific combinations. For the patients with three or more risk factors, one first needs to determine how many risk factors are going to be generated. As can be seen in the left upper corner of Figure 3, a weighted procedure was used to ensure that a low number of risk factors is more plausible.

To determine the thresholds, each of the generated patients makes each kind of call once, i.e., a normal, service and sanitary call, and the patient's responsible nurse makes an assistance and sanitary assistance call. The priorities of other types of calls are not influenced by the risk profile of the patient. For each call, the probabilities are calculated for each of the seven possible priorities. For each priority only a limited number of probabilistic values can be obtained. These are the possible thresholds.

For each combination of possible thresholds, it is determined for each call which priority it gets. If the call has a probability for the highest priority that is higher than or equal to the threshold for the highest priority, the call gets the highest priority. If not, the same condition is checked for high, above normal, below normal, normal, low and lowest priorities and thresholds. If none of these conditions hold, the call gets the priority undetermined. The below normal threshold is checked before the normal one to ensure that the latter is the default priority that calls are assigned to. The low and lowest priority classes are reserved for technical and medical calls.

Using this algorithm, the percentage of calls that are assigned to each priority and the percentage of undetermined calls is calculated for each combination of thresholds. A curve fitting algorithm then determines the appropriate threshold combination by searching the combination for which the percentage deviates least from the ideal distribution, which is determined based on the characteristics of the department, e.g., frequency of calls. For example, the priority distribution 5\%-10\%-25\%$35 \%-25 \%-0 \%-0 \%$, ordered from the highest to lowest priority, reflects a realistic hospital environment. Preference is given to combinations with the least amount of undetermined calls. 


\subsubsection{Nurse call algorithm}

The previous nurse call algorithm, which was detailed in [10], was updated to take the priorities of the calls into account. Additionally, algorithms were devised for the service, sanitary, (sanitary) assistance, technical and medical calls.

The algorithm starts with determining which type of call has been made and acts accordingly. Normal, sanitary, service and (sanitary) assistance calls employ the same basic algorithm. The difference is that for service calls both caretakers and nurses can be called, while for the others only nurses can be assigned. It is also made sure that the nurse, who made the (sanitary) assistance call, is not called again.

This basic algorithm first checks if the responsible nurse or caretaker, further described as helper, is in the vicinity. This responsible helper is called if he/she is busy with a task that has a lower priority than the current call.

If this responsible helper cannot be called, all the helpers, who work on the department where the call originated, are considered. From this group, the helpers, who are not willing or qualified to treat the patient or are not in the vicinity, are removed. Only for calls with the highest or high priority, helpers are considered that are busy with a task with a lower priority. Otherwise, the helpers are never able to finish the work for the patients they are responsible for. For calls with lower priorities, the busy helpers are filtered. From the remaining helpers, the one who has the most characteristics in common with the preferences of the patient is chosen. If there are no characteristics specified, the closest helper is chosen.

If this option still does not offer a solution, the search is widened beyond the scope of the department and helpers in the whole hospital are considered. The selection is similar to the previous paragraph, but busy nurses are never considered.

If the result is empty, this means that there are no available helpers in the direct vicinity. The distance becomes a deciding factor. The closest helper, who is free, willing and qualified, is selected.

If this still does not offer a solution, all the helpers in the hospital are considered and the closest one is called. 
The algorithm has a time-out procedure. If a caregiver has not indicated that he/she is going to handle the call within the time-out time that is specified for this type of call in the ontology, another caregiver is selected by running the algorithm again.

Urgency, medical and technical calls each have their own algorithm. For urgency calls, the priority lies on finding a person who is near instead of free. Therefore, the caregiver closest to the patient, who is member of an urgency team and is not already handling another urgency call, is searched. The urgency team to which this person belongs is called. A time-out procedure is not needed, as urgency calls are always immediately answered.

The algorithms for the technical and medical calls are straightforward because they have a low priority. For medical calls, the doctor responsible for the patient is called. For technical calls, the closest, free technician is called. Both algorithms also have a time-out procedure.

Note that a caregiver is sometimes called while he/she is busy with a task. It is up to this person to decide to interrupt this task or not. In contradiction to the currently used system, the caregiver knows that the new call has a higher priority than this task. Based on these priorities the caregiver can make a more informed decision. If the caregiver decides to answer the call, the system automatically interrupts the task. If the task is a call, another caregiver is searched, using the algorithms above. If it is not a call, the task is added to the list of tasks of this person.

The nurse call algorithm takes into account that patients sometimes hit the call button multiple times before their call is handled. When the algorithm notices that the hit originates from a button, which already launched a call that has not been handled yet, no new nurse is assigned. The nurse, who accepted the call, is alerted that the patient pushed the button again. It was chosen not to increase the priority of the call as this would favor impatient patients. Moreover, it would allow patient to cheat the system.

Finally, this algorithm also controls the nurse call lights and tracks the status of the call. When a call is launched, the status of the call is Active. If the call is made inside a room, the call light outside the door is switched on, giving the caregivers a visual cue that a call has been launched inside this room. The lights give a visual indication to the staff how busy the department is. Buttons also light up in the room to assure the patient that the call was properly placed and registered by the system. The nurse 
call algorithm assigns the call and the assigned nurse receives the call on a smartphone. If the nurse accepts the call, the status changes to Accepted. When the nurse arrives in the room, an identification key is used to $\log$ in. The nurse call algorithm registers that the assigned nurse is present and changes the status of the call to Busy. The lights change color, indicating to other caregivers and visitors that a nurse is present. When the nurse leaves and logs out, the status of the call becomes Finished and the lights switch off. If the call did not originate from inside a room and a nurse is not able to log in when tending to the patient, the nurse can indicate on the smartphone that the call is handled.

\subsection{Evaluation set-up}

To determine the thresholds for the priority assessment algorithm and to evaluate their sensitivity, data was gathered about the risk profile of patients in five nursing departments of Ghent University Hospital [11]. Each department contains at most 30 patients. The data is summarized in Table 1. The risk factor combinations which are deemed more frequent overall or in specific departments are also indicated.

To evaluate the developed probabilistic nurse call algorithms, simulations were performed based on data gathered from three of these departments. They differ in the mobility of the patients. In Deptl, the patients are barely mobile. Most of them are in a coma and those that are awake are attached to a lot of equipment. Dept 2 contains patients that are fairly mobile, but spend most of their time in their room. In Dept3, the patients are quite young, so they move around a lot across the whole department. The most important properties of these departments are summarized in Table 2. For each department, at least three spaces were selected to where patients often travel and the travel time from the respective departments was measured. It was determined how patients divide their time over these spaces. Information about the staff in these departments was also gathered. In Dept1 and Dept3, each nurse is responsible for at most two patients who often lie in adjacent beds. In Dept2, each nurse is responsible for a number of patients depending on the shift and the occupancy rate of the department. This can range from 10 patients per nurse during the night shift, to two patients per nurse during the early shift. They are assigned based on the location of the rooms. Patients in rooms close to each other are assigned to the same nurse. This often leads to an unevenly distributed workload amongst the nurses. 
In none of these departments a patient is ever assigned to more than one nurse at a time. Dept 2 and Dept 3 also employ head nurses, but he/she generally does not answer calls. The walking behavior of the staff was simulated by gathering information about their tasks and the percentage of time they spend on each type of tasks, as discussed in Myny, et al. [14]. Each of the tasks was assigned a priority based on how easily the task can be interrupted.

To simulate the calls, logging information, e.g., frequency and duration, about the calls in the departments was gathered during three weeks. The reasons for the calls are simulated based on the percentages cited by Meade [15], who did an extensive study on the subject. The average time that a nurse spends on handling a task from each category was also determined. Normal and assistance calls can be made in the three departments. Sanitary (assistance) calls can only be made in Dept 2 and Dept3, as the patients in Dept1 are bedridden. Service calls cannot be made as the Ghent University Hospital does not employ caretakers. Because of their low or very high priority, low frequency and simple assignment algorithm, as explained in Section 2.2.2, medical, technical and urgency calls are not simulated.

The currently employed nurse call algorithm in the three departments is visualized in Figure 4. It is important to note that the nurses in Deptl do not receive calls on beepers or portable phone. Consequently, they need to see or hear the call. In the three departments, nurses are sometimes called, who are already busy with a task. They have to decide for themselves whether to interrupt it and remember to return to it. In case the interrupted task is a call, the other patient has to wait until the nurse returns, while the new call could be of lower priority. It is also possible that multiple nurses arrive to handle a call, as multiple nurses are called and one nurse does not know if another nurse will handle it or not.

\subsection{Implementation and simulation set-up}

The oNCS, incorporating the dicussed algorithms, was built as an extension of the Context-Aware Service Platform CASP [16][17]. A detailed description of how this extension was realized can be found in Ongenae, et al. [12]. 
Various methods have been proposed in literature to represent and reason about probabilistic knowledge in ontologies [18][19][20][21][22]. For this research, Pronto [23] was used, which reasons on probabilistic information that is added as annotations in the ontology.

A mobile nurse call application was also developed. It is used by the caregivers to receive, assess and accept, i.e., indicate that they are going to handle, calls. When the patient hits the call button multiple times, this is indicated by the application. A nurse can also use the application to contact the patient, e.g., to request the reason for the call or to give feedback to the patient about the expected arrival time, or other caregivers. This application is further explained in Ongenae, et al. [5].

A realistic day-to-day hospital scenario is simulated. The beds in the departments are occupied averaging around the occupation rate indicated in Section 2.3. The simulations assume that patients possess portable buttons and move around freely and make calls according to a Poisson process with $\lambda=0.000388558$ in Dept $1, \lambda=0.001164021$ in Dept2 and $\lambda=0.000112434$ in Dept3. When this is simulated for the current system, some calls may be impossible to handle, e.g., calls made in the middle of a hallway. The movements of the patients and nurses were determined out of the collected data, as discussed in Section 2.3. Once a patient makes a call, it is assumed that he/she stands still. Nurses receive calls while they are doing their tasks. They interrupt their current task, if the call has a higher or equal priority. They interrupt current calls, if the new call has a higher priority. If the new call does not have a priority, which can occur in the current system, a nurse chooses randomly to interrupt the current task or call. If a nurse has to choose between multiple calls to handle, he/she chooses the one with the highest priority. If they do not have priorities or have the same priority, the closest call is chosen. During the handling of a call, caregivers will launch a (sanitary) assistance call with a probability of $0.02979 \%$ in Dept1, $0.07386 \%$ in Dept 2 and $0.20588 \%$ in Dept3. Patients or nurses who are on the move advance 1 meter in the direction of their goal during each time step. Characteristics of patients and nurses, risk factors of the patients and responsibility of caregivers for particular patients were simulated as indicated in Section 2.3.

Each simulation was performed 30 times for each of the shifts during the weekend and week. The simulations were done on a PC with an Intel Core 2 Duo P8600 processor and 4 gigabyte RAM. 


\section{Results}

\subsection{Threshold parameter exploration}

This section explores the sensitivity and applicability of the thresholds generated by the algorithm detailed in Section 2.2.1. This algorithm was used to randomly generate patients, calls and their accompanying probabilities based on the data gathered about the risk profiles of patients as described in Section 2.3. In total 22,500 calls were simulated and 252,252 possible combinations of thresholds were achieved. To evaluate the fluctuation of the total deviation, the 100 threshold combinations with the smallest total deviation from the ideal distribution, namely $5-10-25-35-25-0-0$, were studied. The $100^{\text {th }}$ one has a deviation of 23.93 and 24.04 in the test and validation group respectively. It can be concluded that the deviation only moderately increases across these 100 threshold combinations.

There were 12 combinations of thresholds which had the smallest total deviation, namely 16.44 and 19.20 in the test and evaluation group respectively, from the ideal distribution. Each of these combinations had no undetermined calls. Combinations of thresholds are preferred which ensure that the highest priority is not assigned too much to calls. This priority is mainly reserved for urgency calls. Six of the 12 threshold combinations only assigned around 5\% of calls to this priority. The difference between these combinations is the threshold for the above normal $(0.21,0.24$ or 0.3$)$ and low $(0$ or 0.2$)$ priorities. As the normal priority class has a threshold of 0 for each of the six combinations, the calls never get the low or lowest priority. So 0 is picked as threshold for the low priority. For the above normal priority, the middle threshold of 0.24 is chosen as this guarantees that it is less sensitive to fluctuations.

Thus, the chosen thresholds are $0.21-0.3-0.24-0-0.05-0-0$, ordered from the highest to lowest priority.

The sensitivity of the chosen thresholds was studied by selecting the threshold for one priority, e.g., the highest, and increasing and decreasing it to the next threshold for this priority, e.g., 0.24 and 0.18 . The fluctuation in deviation for these threshold combinations is illustrated in Figure 5a. The percentage of calls that changes priority by changing the threshold is shown in Figure 5b. The x-axis shows for each priority to which threshold the chosen threshold is increased and decreased, while the 
rest of the thresholds stay the same as in the chosen combination. As the chosen threshold for the normal and low priority is 0 , it can only be increased. The lowest priority had only one threshold possibility, thus it cannot be changed. As can be derived from the graphs, only the normal and below normal priorities are very sensitive to threshold changes. This is because the normal priority is used as the default priority with a threshold of zero. Consequently, the low priority becomes the default, when the threshold of the normal priority is increased. This causes a lot of calls to receive this priority instead of the normal one. If the threshold of the below normal priority is decreased, it becomes zero and this priority becomes the default.

The chosen thresholds were also evaluated by studying which percentage of each type of call and patients with zero, one, two and three or more risk factors are assigned to each priority. The results are illustrated in Figure 6. The normal and sanitary calls mostly get the above normal and normal priorities. A low percentage of these calls gets the below normal priority. Service calls generally get a lower priority. This is realistic as these calls are made for caring tasks. The (sanitary) assistance calls often get the highest or high priority. This is desirable as immediate help is often required. The calls of patients who have no risk factors are assigned the default priority, namely normal, as risk factors are the only criteria used in this initial study to determine the priority. Calls made by patients with one risk factor are distributed equally among all the priorities. In this case, the priority depends on the severity of the risk factor. As the number of risk factors increases, the calls are more likely to receive a higher priority.

\subsection{Simulation Results}

Figure 7 shows for the three departments the number of calls that have a nurse present before a specific time point. Note that the first part of the $\mathrm{x}$-axis goes in jumps of five seconds, while the second part jumps 60 seconds each time.

In Deptl there is no significant difference between the oNCS and current system as a lot of nurses are present who can readily answer calls.

However, in Dept2 and Dept 3 there is a notable difference. In the $o N C S$ only one nurse receives the call, which often has a higher priority than his/her current task. Therefore, the nurse immediately 
answers the call. In the current system, multiple nurses receive the call. They decide if they quit their current task without additional context information. If all the nurses ignore the call, it has to be relaunched, as illustrated by peaks on the graph after the relaunch times, e.g., 180 or 360 seconds. Moreover, the oNCS takes the walking distance into account when assigning a nurse. This explains the difference between Dept 2 and Dept3. The circular design of Dept 3 makes it possible for the nurses to reach rooms faster.

In all three departments, the peak of the oNCS occurs somewhat later, as running the algorithm causes an initial delay.

In Dept1 and Dept2, the tail of the oNCS is very long, as all impossible calls are answered. Most of these calls occur in distant places. This could be compensated by allowing nurses from closer departments to answer these calls.

Figure 8 visualizes the percentage of calls of a particular type that have a nurse present before a specific time point.

In Dept 1, the curves of the assistance calls are comparable between both systems, as these calls generally have a very high priority. The normal calls generally have a nurse present faster in the current system. This can be explained by the high amount of nurses present in the department. In the current system every nurse that sees the call can handle it. In the oNCS only one nurse receives the call and is expected to handle it. This nurse might not be the closest, free nurse, as the algorithm prefers the responsible nurse.

In Dept2, the sanitary assistance calls have a nurse present within 15 seconds in both systems. However, the $o N C S$ performs much better than the current system for the other types of calls. Due to the lower amount of nurses, an occupied nurse is often called. The oNCS prefers occupied nurses who are busy with a task that has a lower priority than the call. This is often the case for assistance and sanitary calls. As the nurse is aware of the call's higher priority, he/she immediately handles it. In the current system, the nurse has to decide at random to interrupt the task.

In Dept3, the curves of the assistance calls are quite different. The curve of the oNCS rises faster but then slows down and has a much longer tail. Sanitary calls on the other hand are handled very fast in the oNCS. However, only a few sanitary calls were simulated. In Dept3, a toilet is available in the 
room. Calls made inside the room were registered as normal, as it is impossible to track with enough accuracy if the patient is on the toilet. Only calls made inside designated sanitary areas were registered as sanitary. The normal calls generally have a nurse present faster in the oNCS. One type of call does not get answered notably faster than another kind in Dept3. As patients in this department have a wide variety of risk factors, a normal call can have the same priority as for example an assistance call. Consequently, the assistance call is not always handled faster than the normal one.

The number of calls with a particular priority that have a nurse present before a specific time point are visualized in Figure 9.

In all departments, the highest priority is assigned the least, which is desirable as it is mostly reserved for urgency calls. In Dept1, the above normal priority is assigned the most, as Dept1 contains a considerable amount of patients with two or more risk factors. In Dept2 and Dept3, the below normal priority is assigned the most as these departments contain a lot of patients without risk factors.

In the oNCS, the highest priority calls are handled the fastest in all the departments. In Dept 3 two assistance calls with the highest priority were made while all the other nurses were away, causing these calls to be handled quite slowly. The below normal, normal and above normal priority calls are handled somewhat slower than the highest priority calls. In Dept3, calls with these priorities also have the same worst-case time. In Dept2, the tails of the worst-case time are in the correct order: first the above normal, than the normal and finally the below normal priority calls. However, in Deptl the above normal priority calls have the highest worst-case time, as many calls are assigned this priority. If a lot of these calls are made simultaneously, they interfere with each other.

It is clear that the current system does not take the priorities into account. Even the highest priority calls need to be relaunched a couple of times. The above normal, normal and below normal priority calls have the same trend in Dept2 and Dept3. However, the improvement of the oNCS compared to the current system is not as notable in Dept1. Although, the below normal priority calls are generally handled slightly faster than the normal priority calls in the current system, the rest of the graph is quite similar to the graph of the oNCS. This is caused by the high amount of nurses in the department. 
Finally, the distribution of calls amongst the nurses is illustrated in Table 3 . The first column indicates the number of nurses in the department during the simulation. The maximum and minimum percentage of calls handled by a nurse during a shift, how many nurses handle zero calls during a shift and the standard deviation between the percentage of calls that nurses handle and the mean are indicated.

The $o N C S$ leads to a slightly better workload distribution in Dept 1 and Dept2 and a much better result in Dept3. There are less nurses that get the extreme percentages and the highest percentage of calls that a nurse can be assigned is also lower. In Dept3, the improvement is especially notable in case there are only two nurses in the department. In the oNCS, all the nurses get calls, while in the current system only one of the nurses handles the calls.

\subsection{Performance results}

To evaluate the scalability of the probabilistic reasoning, reasoning is performed on an ontology with gradually increasing number of probabilistic statements.

As visualized in Figure 10, time gradually increases and starts exploding around 20 probabilistic statements. At 24 statements, the performance becomes unacceptable, namely around 30 or 40 minutes. The scalability is thus an issue [24]. However, performing the probabilistic reasoning on 12 or less probabilistic statements is always below 4 seconds, which is acceptable for our application.

The following optimization was employed to speed up the probabilistic reasoning. First, during downtime, the probabilities for each patient that he/she is a high, medium or low risk patient are calculated and stored as known facts in the ontology. This procedure does not have to be repeated often as risk factors do not change often for a patient during his/her stay at the hospital. Next, when a call is made, all the probabilistic statements needed to calculate the priority of this call are extracted from the ontology. This will be at most 12 statements, namely the statements about the probabilistic assignment of this patient to the risk groups and the generic probabilistic assignment of this kind of call to the priority groups.

Once the priority is determined, a suitable nurse is notified within $50.3 \mathrm{~ms}$ on average, which is a negligible delay. A more thorough discussion of this result can be found in Ongenae, et al. [12]. 


\section{Discussion}

First, the oNCS leads to measurable improvements in the manner nurses are assigned to calls by taking the context information into account.

The time to intervention is an important parameter as it is essential to quickly assess the situation. The health of the patient could be compromised in which case fast intervention is of paramount importance. Some countries even outline guidelines that stipulate that the time to intervention should be within 3 minutes for urgency calls and within 5 minutes for other calls. The time to intervention is also correlated with patient [25] and caregiver's job satisfaction as a quicker time to intervention leads to fewer calls being assigned again because of a time-out and thus to less unnecessary work interruptions. Work interruptions are one of the main factors of cognitive fatigue and errors and have a significant impact on the workload distribution and performance [26].

The oNCS generally achieves a better time to intervention and workload distribution than the current system. Moreover, calls with a higher priority are generally handled faster. (Sanitary) assistance calls are also handled faster than normal and sanitary calls. This is often not the case in the current system. In the $O N C S$, only one caregiver is called to handle a call. The algorithm takes the distance and the current tasks of the caregivers and their priorities into account. When a caregiver receives a call while performing a task, this new call generally has a higher priority. The caregiver will thus more likely interrupt this task because of this higher priority and because he/she knows he/she is the most appropriate person to handle the call at this moment. The caregiver can also request additional information about the call, the condition of the patient and other caregivers, e.g., location or current tasks, on the smartphone to determine whether to answer it. He/she can directly contact the patient or caregiver to assess the reason and whether he/she needs to bring equipment or medication. If he/she accepts the call on the smartphone, it is also expected that he/she will tend to it. Other nurses are not aware of the call unless they see the light above the room of the patient. When a task is interrupted, the nurse does not have to remember to return to it. The oNCS does this for the nurse. This leads to fewer forgotten tasks and less work pressure on the staff.

The oNCS thus also requires a change in mentality from the caregivers pertaining to how calls are handled. The nurses need to trust that the system has assigned the nurse who is most appropriate at 
that moment. They thus need to let go of the instinct to walk into a room because the light is burning. This allows them to focus on their, possibly more urgent, current task. However, they are able to walk in and start tending to the patient if they want to. The mobile application does not allow seeing an overview of all the current calls and which nurses are assigned to them. So it is difficult to get in touch with the assigned nurse to notify that somebody is already handling the call. A page could be added to the mobile application where all the current calls are listed with their status and who has been assigned to them. This could easily be achieved as all this information is readily available in the ontology.

In contradiction, in the current nurse call system unnecessary nurses are often called, resulting in multiple nurses (or no nurse at all) arriving at a call. This leads to interruptions of other tasks and unnecessary walked miles. Moreover, they have to decide without context information, e.g., priority of call or availability of other staff, whether to rush to the call or first finish their current task. If they interrupt their task, they have to remember themselves to return to it.

As the results of the simulations of Dept2 and Dept 3 clearly show, the benefit of the oNCS with probabilistic priority assessment is biggest in nursing units with a small number of caregivers, who answer calls, compared to the number of patients and a high degree of patient heterogeneity, meaning that the patients have very different risk factors and the department thus contains low, medium and high risk patients.

The first is clearly illustrated by the simulations in Dept1. In this department there is one nurse per two patients. Up to six beds are also grouped in the same space. Consequently, there is always a nurse close to the patient making the call. Moreover, this nurse can also easily view and talk to the patient while he/she is busy with another task. The walking distance to the patient making the call and the need to interrupt tasks is far less in Dept1 than in Dept2 and Dept3. This conclusion is also reflected by the workload distribution, which stays more or less the same when comparing the oNCS to the current system.

The second is caused by the fact that patients with a similar risk profile will make calls of similar priority. This is clearly illustrated by the simulations of Dept 1 where most of the patients have a lot of risk factors. Consequently, they are classified as high risk patients. Thus if patients with the same risk factors (or similar ones) make calls, the priority of these calls only probabilistically depends on the 
type of call. As no differentiation can be made based on the priority of the call, the nurse call system will try to find caregivers who are free and/or close to handle the call. Thus, in these cases the probabilistic priority assessment algorithm contributes little to the nurse call assignment. However, as previously discussed, the oNCS still offers a lot of other benefits that are still applicable in such departments.

The issue could be addressed in future iterations of the system by letting the priority of the call depend on more or other information than just the risk factors of the patient and the type of call, e.g., monitored medical parameters of the patient, such as high temperature or blood pressure, or profile information, such as, frequent caller. Moreover, before the oNCS is installed in a nursing unit, it needs to be evaluated if the probabilistic priority assessment, which requires considerable computation cost, will have significant impact on the nurse call assignment, i.e., enough heterogeneity amongst the patient and a small number of nurses compared to the number of patients. If not, the oNCS could be installed with a simpler nurse call algorithm, thus offering all the benefits of the context-awareness and portable buttons without the computational cost of the probabilistic priority assessment.

Second, the performance of the novel nurse call algorithm is also very good, as a suitable nurse is notified within $50.3 \mathrm{~ms}$ on average, which is a negligible delay. The system scales up to at least 30 patients and 20 nurses. Thus, a lot of profile information can be retained without decreasing the performance of the system. Moreover, at least 30 calls can be made simultaneously without influencing the performance.

Third, the portable buttons improve the mobility and the safety of the patients. Patients can walk around freely and make calls. It can be derived from the simulations that it often occurs that patients need to make calls in remote areas such as smoking areas or the restaurant However, patients could potentially exploit the system as they can call a nurse from anywhere in the hospital even for trivial requests. This could increase the walking distance and workload of the nurses.

It is however important to note that the developed oNCS does not necessarily need to be combined with mobile call buttons to offer advantages. Even when fixed buttons are used, the oNCS offers improvements compared to the traditional systems as its nurse call algorithm is more dynamic by taking a plethora of context information into account, as already discussed. 
However, our study is limited by the fact that the probabilities in the ontology were only determined by domain experts. Collecting data out of which these probabilities could be determined or with which the probabilities could be validated, requires a very extensive study. The study would for example require that nurses note down for each call they handled which priority it had and for which reason the call was made. This requires a significant effort from the already time constrained caregivers. This study was thus not conducted as the goal was to first validate whether incorporating the probabilistic priority assessment in the oNCS would offer significant benefits. Basing this study on probabilities determined by an expert panel with years of experience in the field gives us a good idea of the impact of the $o N C S$.

However, we do acknowledge that requiring these probabilities as input for the oNCS could prove cumbersome to determine or assess by the departments and hospitals where the oNCS would be deployed. Therefore, research is on-going on extending the $o N C S$ with an autonomic, self-learning component [27]. This component uses data collected about patients and logged by the oNCS about its usage to gradually adapt the probabilities such that an optimal configuration of the oNCS is obtained for this department or hospital.

\section{Conclusion}

This article described an extension of the Ontology-based nurse call system (oNCS) that supports a more sophisticated nurse call algorithm by dynamically assigning priorities to calls based on the risk factors of the patients and the type of call. The benefits of this novel oNCS were illustrated with extensive simulations about data collected from three departments of the Ghent University Hospital. The oNCS significantly improves the assignment of nurses to calls. Calls generally have a nurse present faster, the workload-distribution amongst the nurses improves and the priorities and kinds of the calls are taken into account. The execution time of the nurse call algorithm is negligible.

\section{Acknowledgements}

We would like to acknowledge that this research was supported by the head nurses Annemie Danhieux and Johan Van Daele and the assistant head nurse Carine Gevaert. Special thanks also goes out to my 
colleague Matthias Strobbe for supporting the implementation in the CASP platform. Finally, we would like to acknowledge the people behind Pronto and especially Pavel Klinov for always being open to questions and discussions.

Femke Ongenae is funded by the Institute for the Promotion of Innovation by Science and Technology in Flanders (IWT).

\section{References}

[1] Zuker, A, Heart, T, Parmet Y, Pliskin, N, Pliskin, JS. Electronic notifications about drug substitutes can change physician prescription habits: a cross-sectional observational study. Med Decis Making. 2011; 31 (3): $395-404$.

[2] Naik, AD, Singh, H. Electronic health records to coordinate decision making for complex patients: What can we learn from Wiki? Med Decis Making. 2010; 30(6): 722-731.

[3] Bottieau, E, Moreira, J, Clerinx, J, Colebunders, R, Van Gompel, A, Van den Ende, J. Evaluation of the GIDEON expert computer program for the diagnosis of imported febrile illnesses. Med Decis Making. $2008 ; 28(3): 435-442$.

[4] Ongenae F, Bleumers L, Sulmon N, Verstraete M, Van Gils M, Jacobs A, et al. Participatory design of a continuous care ontology: towards a user-driven ontology engineering methodology. In: Filipe J, Dietz JLG, editors. KEOD 2011: Proceedings of the International Conference on Knowledge Engineering and Ontology Development; 2011 October 26-29; Paris, France. SciTePress. p. 81-90.

[5] Ongenae F, Duysburgh P, Verstraete M, Sulmon N, Bleumers L, Jacobs A, et al. User-driven design of a context-aware application: an ambient-intelligent nurse call system. In: U-CDPHA 2012: Proceedings of the User-Centered Design of Pervasive Healthcare Applications Workshop of the 6th International Conference on Pervasive Computing Technologies for healthcare; 2012 May 21-24; San Diego, CA, USA. Washington:IEEE Computer Society. p. 205-210.

[6] Wachter GW. Hospitals Unplugged: The Wireless Revolution Reaches Healthcare. Telemedicine Information Exchange [serial online]. 2001 [cited 2010 July 28]. Available from: http://tie.telemed.org/articles/article.asp?path=articles\&article=hospitalsUnplugged_gw tie01.xml.

[7] Miller ET, Deets C, Miller R. Nurse call and the work environment: lessons learned, J Nurs Care Qual. 2001; 15(3): 7-15. 
[8] Linden L, English K. Adjusting the cost-quality equation: Utilizing work sampling and time study data to redesign clinical practice. J Nurs Care Qual.1994; 8(3): 34-42.

[9] Gruber T. A translation approach to portable ontology specifications. Knowl Acquis, 1993; 5(2): 199-220.

[10] Ongenae F, Strobbe M, Hollez J, De Jans G, De Turck F, Dhaene T, et al. Design of a semantic personoriented nurse call management system. IJWGS. 2008; 4(3): 267-283.

[11] Ghent University hospital. Available from: http://www.healthcarebelgium.com/index.php?id=uzgent [cited 2010 July 28].

[12] Ongenae F, Myny, D, Dhaene, T, Defloor, T, Van Goubergen, D, Verhoeve, P, et al. An ontology-based nurse call management system (oNCS) with probabilistic priority assessment. BMC Health Services Research. 2011; 11:26.

[13] Sirin E, Parsia B, Grau BC, Kalyanpur A, Katz Y. Pellet: A practical OWL-DL Reasoner. J Web Semant. 2007; 5(2): 51-53. Available from: http://pellet.owldl.com/ [cited 2010 July 28].

[14] Myny D, Van Goubergen D, Limère V, Gobert M, Verhaeghe S, Defloor T. Determination of standard times of nursing activities based on a Nursing Minimum Dataset. J. Adv. Nurs. 2010; 66(1): 92-102.

[15] Meade CM, Bursell AL, Ketelsen L. Effects of Nursing Rounds on Patients' Call Light Use, Satisfaction and Safety. Am J Nurs. 2006; 106(9), 58-70.

[16] Strobbe M, Hollez J, De Jans G, Van Laere O, Nelis J, De Turck F, et al. Design of CASP: an open enabling platform for context aware office and city services. In: Pfeifer T, Strassner J, Dobson S, editors. Managing Ubiquitous Communications and Services. MUCS 2007: Proceedings of the $4^{\text {th }}$ International IEEE Workshop on Management of Ubiquitous Communications and Services; 2007 May 27; Munich, Germany. Berlin: Multicon Verlag; 2007. p. 123-142.

[17] Strobbe M, De Jans G, Hollez J, Goeminne N, Dhoedt B, De Turck F, et al. Design of an open contextaware platform enabling desk sharing office services. In: Arabnia HR, editor. Pervasive Systems Computing. PSC 2006: Proceedings of the International Conference on Pervasive Systems \& Computing; 2006 June 26-29; Las Vegas, Nevada, USA. CSREA Press; 2006. p. 135-141.

[18] Yang Y, Calmet J. OntoBayes: An Ontology-Driven Uncertainty Model. In: Mohammadian M, editor. IAWTIC 2005: Proceedings of the International Conference on Intelligent Agents, Web Technologies and Internet Commerce; 2005 November 28-30; Vienna, Austria. Washington: IEEE Computer Society. p. 457 463. 
[19]Ding Z, Peng Y. A Probabilistic Extension to Ontology Language OWL. In: HICSS-37 2004: Proceedings of the $37^{\text {th }}$ annual Hawaii International Conference on System Sciences; 2004 January 5-8; Big Island, Hawaii. Washington: IEEE Computer Society.

[20]Laskey KB. MEBN: A Language for First-Order Bayesian Knowledge Bases. Artif Intell. 2008; 172(2-3): 140-178.

[21] Baader F, Calvanese D, McGuinness D, Nardi D, Patel-Schneider P. The description logic handbook: theory, implementation and applications. Cambridge: The Press Syndicate of the University of Cambridge; 2003.

[22] Lukasiewicz T. Probabilistic Description Logics for the Semantic Web. INFSYS Research Report 1843-0605. Wien, Austria: Technical University of Wien, Institute for Information Systems; 2007.

[23] Klinov P. Pronto: A Non-monotonic Probabilistic Description Logic Reasoner. In: Bechhofer S, Hauswirth M, Hoffmann J, Koubarakis M, editors. The Semantic Web: Research and Applications. ESWC 2008: Proceedings of the $5^{\text {th }}$ European Semantic Web Conference; 2008 June 1-5; Tenerife, Canary Islands, Spain. Berlin: Springer. p. 822-826. Available from: http://pellet.owldl.com/pronto [cited 2010 July 28].

[24] Klinov P, Parsia B. Optimization and Evaluation of Reasoning in Probabilistic Description Logic: Towards a Systematic Approach. In: Sheth A, Staab S, Dean M, Paolucci M, Maynard D, Finin T, et al., editors. Lecture Notes in Computer Science. ISWC 2008: Proceedings of the $7^{\text {th }}$ International Semantic Web Conference; 2008 October 26-30; Karlsruhe, Germany. Berlin: Springer. p 213-228.

[25] Tzeng, H, Ronis, DL, Yin, C. Relationship of actual response time to call lights and patient satisfaction at 4 US hospitals. J Nurs Care Qual. 2012; 27(2): E1-E8.

[26] McGillis, L, Pedersen, C, Hubley, P, Ptack, E, Hemingway, A, Watson, C, et al. Interruptions and Pediatric Patient Safety. Journal of Pediatric Nursing. 2010; 25(3): 167-175.

[27] Ongenae, F, Claeys, M, Dupont, T, Kerckhove, W, Verhoeve P, Dhaene T, et al. A probabilistic ontologybased platform for self-learning context-aware healthcare applications. Expert Systems with Applications. 2013; 40(18): 7629-7646. 


\section{Tables}

\begin{tabular}{|c|c|c|c|c|c|c|}
\hline \multicolumn{2}{|l|}{ Departments: } & \multirow{2}{*}{$\begin{array}{c}\text { Dept1 } \\
0\end{array}$} & \multirow{2}{*}{$\begin{array}{r}\text { Dept2 } \\
10\end{array}$} & \multirow{2}{*}{$\begin{array}{r}\text { Dept3 } \\
15\end{array}$} & \multirow{2}{*}{$\begin{array}{r}\text { Dept4 } \\
4\end{array}$} & \multirow{2}{*}{$\begin{array}{r}\text { Dept5 } \\
0\end{array}$} \\
\hline Number of patients & 0 risk factors & & & & & \\
\hline with & 1 risk factor & 9 & 10 & 10 & 10 & 2 \\
\hline & 2 risk factors & 6 & 8 & 4 & 12 & 8 \\
\hline & $>2$ risk factors & 5 & 2 & 1 & 4 & 20 \\
\hline \multirow{15}{*}{$\begin{array}{l}\text { Risk factor weights } \\
\text { (\%) }\end{array}$} & High age (a) & 36 & 50 & 0 & 15 & 55 \\
\hline & Diabetes (b) & 8 & 10 & 20 & 2 & 5 \\
\hline & Heart disease (c) & 10 & 3 & 19 & 2 & 5 \\
\hline & High fall risk (d) & 2 & 5 & 0 & 13 & 5 \\
\hline & Neurologic problem (e) & 7 & 3 & 10 & 16 & 5 \\
\hline & Tracheotomy (f) & 5 & 10 & 5 & 5 & 2 \\
\hline & COPD (g) & 10 & 3 & 1 & 2 & 5 \\
\hline & Paraplegia (h) & 2 & 3 & 2 & 16 & 3 \\
\hline & Pneumonia (i) & 10 & 3 & 30 & 2 & 5 \\
\hline & Disoriented/ confused (j) & 5 & 5 & 0 & 2 & 5 \\
\hline & Gastric Bleeding within $48 \mathrm{~h}(\mathrm{k})$ & 3 & 3 & 1 & 2 & 2 \\
\hline & Transferred from ICU (1) & 0 & 1 & 10 & 15 & 2 \\
\hline & $\begin{array}{l}\text { Transferred from ICU within } 72 \mathrm{~h} \\
\text { (m) }\end{array}$ & 0 & 0 & 5 & 0 & 0 \\
\hline & Reanimated (n) & 2 & 1 & 2 & 8 & 1 \\
\hline & Reanimated within $72 \mathrm{~h}(\mathrm{o})$ & 2 & 0 & 0.05 & 4 & 0 \\
\hline Combinations & & $\begin{array}{l}\mathrm{f} \text { and } g \\
\mathrm{a} \text { and } \mathrm{c}\end{array}$ & $\begin{array}{l}\text { a and d } \\
b \text { and j }\end{array}$ & None & $\begin{array}{l}d \text { and } h \\
j \text { and } n \\
j \text { and } o\end{array}$ & $a$ and $d$ \\
\hline Overall Combinations & & f and $\xi$ & and $\mathrm{j}, \mathrm{b}$ & $\mathrm{d} \mathrm{j}, \mathrm{e} a$ & 1 and $\mathrm{f}$ & \\
\hline
\end{tabular}

Table 1: The specifics of the five departments for the threshold research 


\begin{tabular}{|c|c|c|c|c|c|}
\hline \multicolumn{3}{|c|}{ Properties } & Dept1 & Dept2 & Dept3 \\
\hline \multicolumn{3}{|l|}{ Mobility } & Low & Medium & High \\
\hline \multicolumn{3}{|l|}{ Nr. of beds } & $\begin{array}{l}22 \text { adults, } \\
6 \text { children }\end{array}$ & 26 & 18 \\
\hline \multicolumn{3}{|c|}{ Occupation rate } & $\begin{array}{c}116,15 \%, \\
87,58 \%\end{array}$ & $84,62 \%$ & $70 \%$ \\
\hline \multicolumn{3}{|c|}{ Most visited spaces by patients } & $\begin{array}{c}\text { Angiography room, } \\
\text { CT scanner, } \\
\text { Radiology } \\
\text { department, } \\
\text { Recovery } \\
\end{array}$ & $\begin{array}{l}\text { Smoking area } \\
\text { outside, } \\
\text { Cafeteria, } \\
\text { CT scanner }\end{array}$ & $\begin{array}{c}\text { Surgery, } \\
\text { Ultrasound, } \\
\text { Receiving electrodes } \\
\text { to monitor sleep }\end{array}$ \\
\hline \multirow{6}{*}{$\begin{array}{l}\text { Nr. of } \\
\text { nurses }\end{array}$} & \multirow{2}{*}{$\begin{array}{l}\text { Early } \\
\text { shift }\end{array}$} & Week & 14 & 5 & 3 \\
\hline & & Weekend & 14 & 4 & Closed \\
\hline & \multirow{2}{*}{$\begin{array}{l}\text { Late } \\
\text { shift }\end{array}$} & Week & 14 & 4 & 2 or 3 \\
\hline & & Weekend & 14 & 3 & Closed \\
\hline & \multirow{2}{*}{$\begin{array}{l}\text { Night } \\
\text { shift }\end{array}$} & Week & 14 & 1 or 2 & 1 \\
\hline & & Weekend & 14 & 1 or 2 & Closed \\
\hline \multirow{4}{*}{$\begin{array}{l}\text { Nr. of head } \\
\text { nurses } \\
\text { (who } \\
\text { answer } \\
\text { calls) } \\
\end{array}$} & \multicolumn{2}{|l|}{ Week } & $1 \& 2$ assistants & I & I \\
\hline & \multicolumn{2}{|c|}{ Weekend } & 1 assistant & I & l \\
\hline & & & & & \\
\hline & & & & & \\
\hline
\end{tabular}

Table 2: The specifics of the 3 departments of Ghent University Hospital used for the simulations of the probabilistic nurse call system 


\begin{tabular}{|c|c|c|c|c|c|c|c|c|c|}
\hline \multirow[t]{2}{*}{ Department } & \multirow[t]{2}{*}{$\begin{array}{l}\text { Nr. of nurses } \\
\text { in the department }\end{array}$} & \multicolumn{4}{|c|}{$\begin{array}{l}\text { Workload distribution } \\
\text { current system }\end{array}$} & \multicolumn{4}{|c|}{$\begin{array}{l}\text { Workload distribution } \\
\text { oNCS }\end{array}$} \\
\hline & & Max. & Min. & \# 0\% & Std. Err. & Max. & Min. & \# $0 \%$ & Std. Err. \\
\hline \multirow[t]{2}{*}{ Dept1 } & 15 & 44.4 & 0 & 241 & 9.0 & 40 & 0 & 206 & 7.6 \\
\hline & 17 & 42.9 & 0 & 265 & 7.5 & 40 & 0 & 252 & 7.3 \\
\hline \multirow[t]{5}{*}{ Dept2 } & 1 & 100 & 100 & 0 & 0 & 100 & 100 & 0 & 0 \\
\hline & 2 & 71.0 & 29.0 & 0 & 11.9 & 57.1 & 42.9 & 0 & 5.1 \\
\hline & 3 & 58.3 & 12.1 & 0 & 12.7 & 61.0 & 7.0 & 0 & 14.7 \\
\hline & 4 & 62.5 & 0 & 1 & 12.3 & 50 & 0 & 1 & 10.2 \\
\hline & 5 & 54.8 & 0 & 2 & 12.6 & 42.5 & 2.6 & 0 & 7.9 \\
\hline \multirow[t]{4}{*}{ Dept3 } & 1 & 100 & 100 & 0 & 0 & 100 & 100 & 0 & 0 \\
\hline & 2 & 100 & 0 & 2 & 47.4 & 75 & 25 & 0 & 17.0 \\
\hline & 3 & 100 & 0 & 20 & 41.3 & 100 & 0 & 10 & 32.6 \\
\hline & 4 & 100 & 0 & 17 & 32.0 & 100 & 0 & 8 & 23.2 \\
\hline
\end{tabular}

Table 3: Shows the distribution of calls amongst the nurses for each department and for each possible number of nurses in this department. The maximum and minimum percentage of calls assigned to a nurse and the number of nurses who got no calls is shown. Finally, the standard error is given between the percentage of calls that nurses handle and the mean. The mean is the ideal percentage of calls that a nurse should handle. 


\section{Figure Legends}

Figure 1: The ontology modeling 1) the profile information of nurses and patients and 2) the types of calls that can be launched and who can handle them. The squares symbolize concepts. The black, dashed arrows represent subclass relationships (is a). The colored arrows indicate relationships between concepts.

Figure 2: Overview of the probabilistic priority assessment algorithm and context-aware nurse call algorithm

Figure 3: Flow of the generation of a test or validation group of each department used to determine the thresholds for the priority assessment algorithm

Figure 4: Flow chart of the currently employed nurse call algorithms in the three simulated departments

Figure 5: Study of the sensitivity of the threshold parameters: a) Fluctuation of the deviation b) The percentage of calls that changes priority category

Figure 6: The percentage of each kind of call and the percentage of patients with $0,1,2$ and 3 or more risk factors that are assigned to each priority category, both for the test group as the validation group Figure 7: The number of calls (y-axis) that have a nurse present before a specific time point ( $\mathrm{x}$-axis, in seconds)

Figure 8: The percentage of calls (y-axis) of a particular kind that have a nurse present before a specific time point ( $\mathrm{x}$-axis, in seconds)

Figure 9: The number of calls (y-axis) with a particular priority that have a nurse present before a specific time point (x-axis, in seconds)

Figure 10: The performance results of the probabilistic reasoning tasks 


\section{Priority Assessment algorithm}

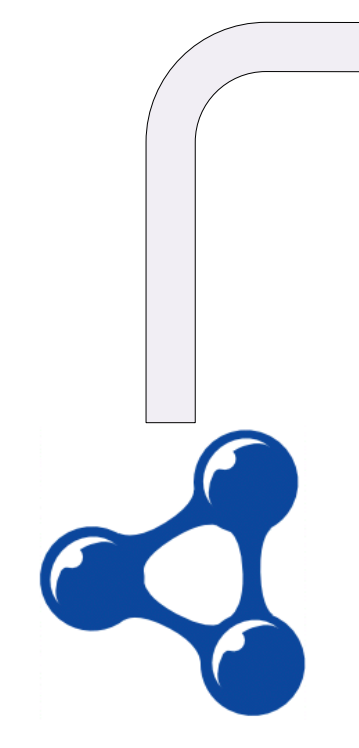

Ontology modeling patient $\&$ staff profiles, calls and tasks

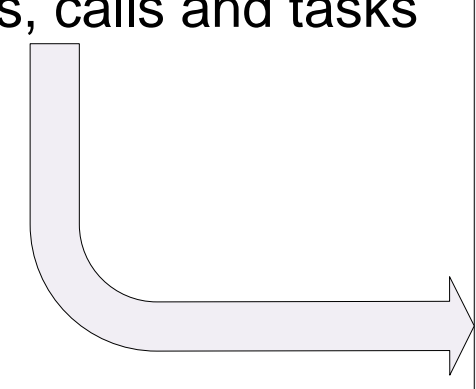

Risk assessmen

Risk factors? $\mathrm{P}$ (High Risk Patient | Heart Disease) Heart disease? $\mathrm{P}$ (Medium Risk Patient | Heart Disease)

Reanimated? $\mathrm{P}$ (Low Risk Patient | Heart Disease)

High age? $\mathrm{P}$ (High Risk Patient | Reanimated)

Patient P(High Risk Patient |Reanimated)

Launches call or call is launched for patient by staf

\section{Nurse call algorithm}

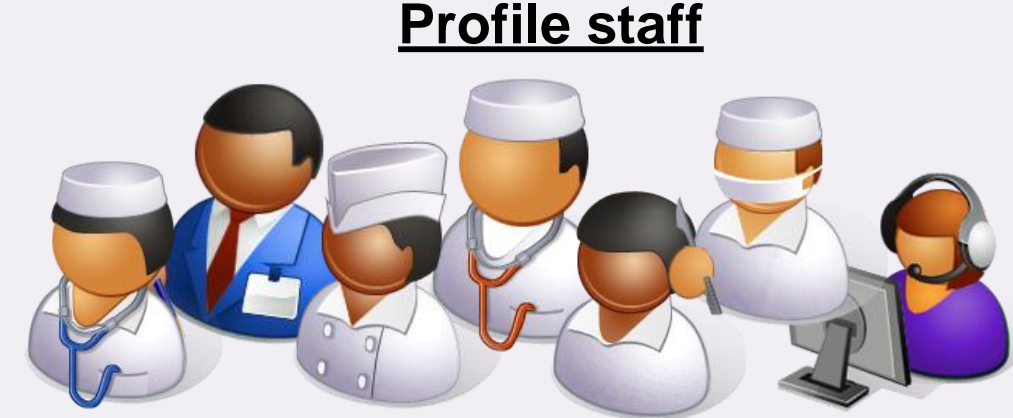

Competences

Characteristics

$$
\begin{aligned}
& \text { Trust relationship } \\
& \text { with patients }
\end{aligned}
$$

Role

Location

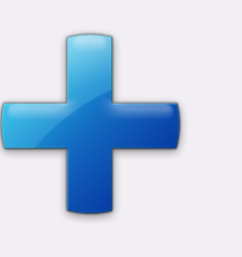

Priority assessment

$P$ (High Priority | High Risk Patient $\cap$ Normal Call)

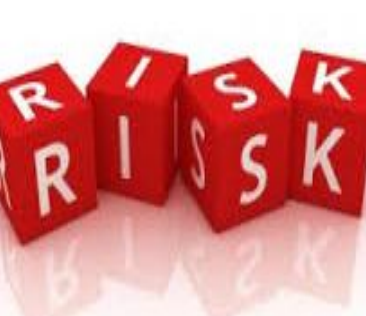

$\mathrm{P}$ (High Priority | Medium Risk Patient $\cap$ Assistance Call)

$P($ Normal Priority | Low Risk Patient $\cap$ Assistance Call)

$\mathrm{P}$ (Normal Priority | High Risk Patient $\cap$ Normal Call)

Type of call

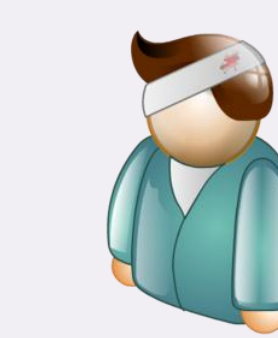

Location

Department

Characteristics
10

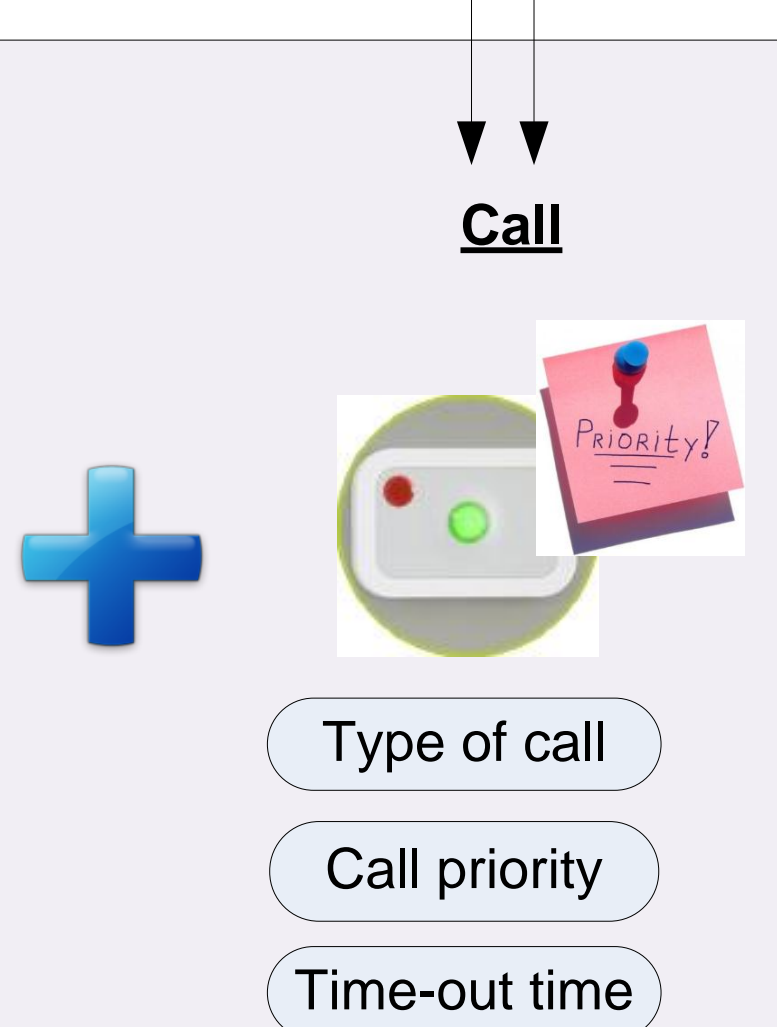

Task

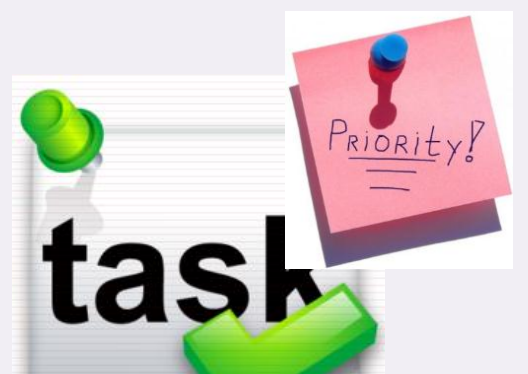

Current task

Task priority
Priority

Thresholds

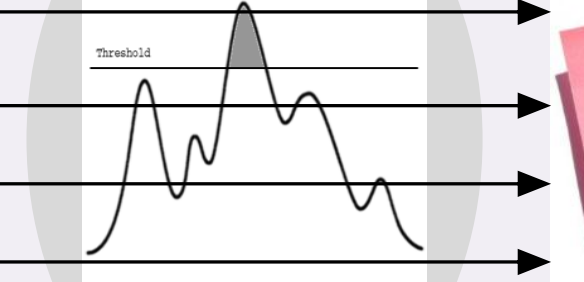

$\ldots$

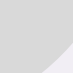


COMBS: Generally important combinations

NAME: Department name

ZERORISKS: Nr. Of patients with 0 risk factors

ONERISK: Nr. Of patients with 1 risk factor TWORISKS: Nr. Of patients with 2 risk factors

MORERISKS: Nr. Of patients with 3 or more risk factors

WEIGHTS: risk factor weights

DEPTCOMBS: important combinations in this department

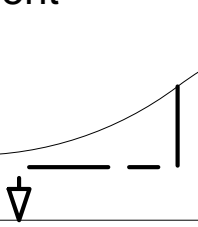

Determine highest weight $(\mathbf{H W})$ in the department<smiles>[Te]</smiles>

Select a risk

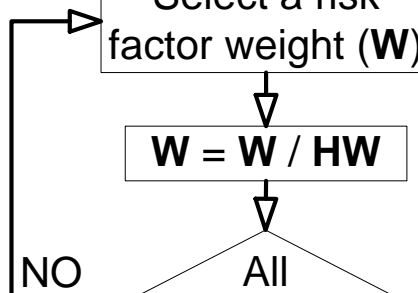

NO

weights rescaled

to interval

$[0,1]$

YES $\rightarrow$

Generate patient

$\longrightarrow$ with 0 risk factors

NO

All ZERORISKS patients generated?

YES $\nabla$

Execute flow

A with $\mathbf{N}=1$

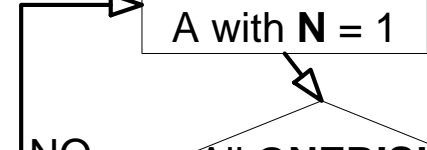

NO All ONERISK

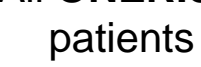

generated?

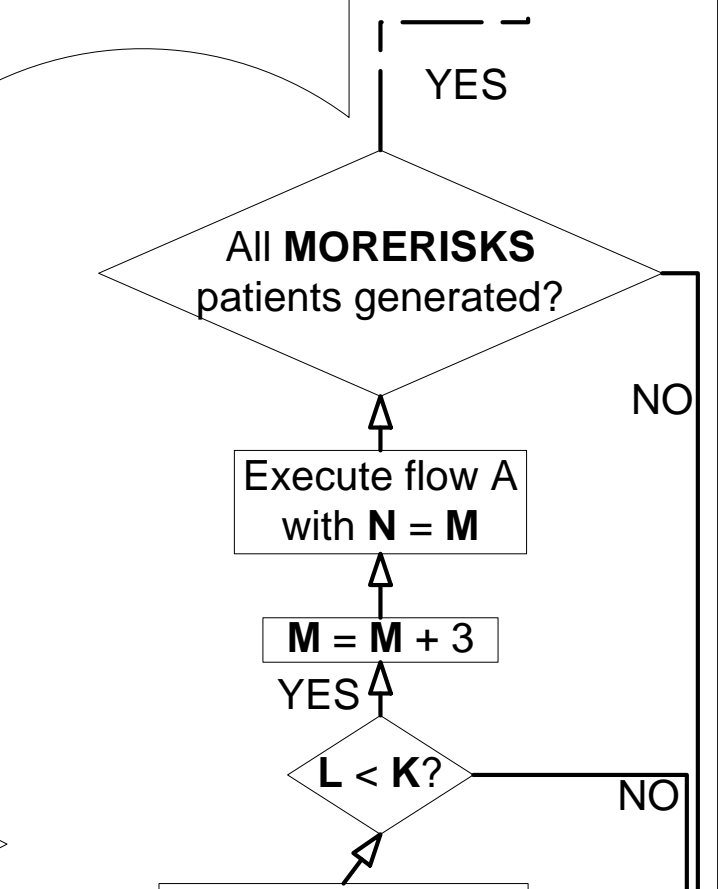

Pick random

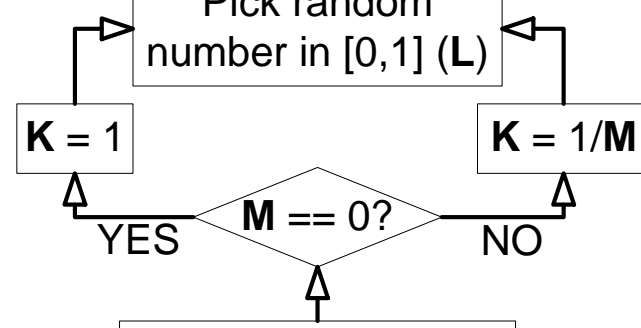

Pick random number in $[0,10](\mathbf{M})<$ YES 4

All TWORISKS

patients generated?

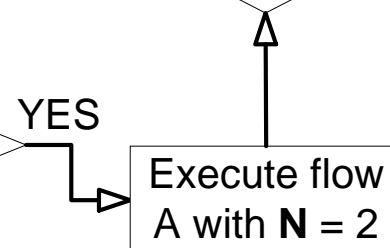

\section{Flow B}

\section{Input:}

NAME: Department name

WEIGHTS: risk factor weights

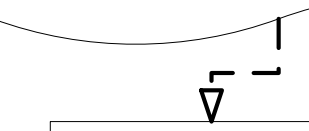

Pick random risk factor (RISK)

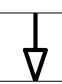

Pick random number in $[0,1]$
Output:

Testset of 30

patients for this

department

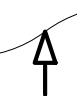

Flow A

Input:

COMBS: Generally important combinations

NAME: Department name

WEIGHTS: risk factor weights

DEPTCOMBS: important combinations in

this department

$\mathrm{N}$ : Nr. Of risk factors that have to be generated

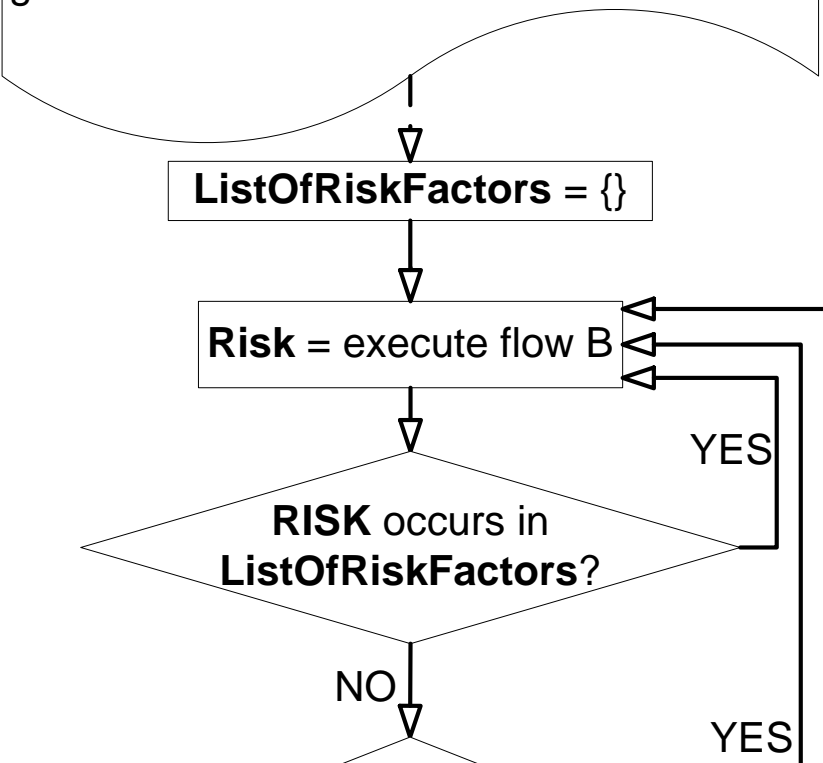

RISK is sub - or super-

class of one of the risks in

ListOfRiskFactors?<smiles>N[Te]CCC[Te]</smiles>

NO

RISK occurs

in COMBS?

YES I

Double the weights of all the risk

factors that have a combination

with this RISK in COMBS

$$
\text { i }
$$

RISK occurs in

DEPTCOMBS?

NO

YES $\nabla$

Double the weights of all the risk factors that have a combination with this RISK in DEPTCOMBS $\nabla$

Add RISK to ListOfRiskFactors $\triangleleft$

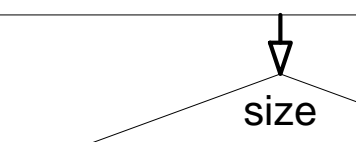

ListOfRiskFactors

NO

$<\mathbf{N}$ ?

YES $\nabla$

Generate patient with all the risk factors in ListOfRiskFactors RISK $\nabla$

\section{Output:}

Generated patient with $\mathbf{N}$ risk factors 

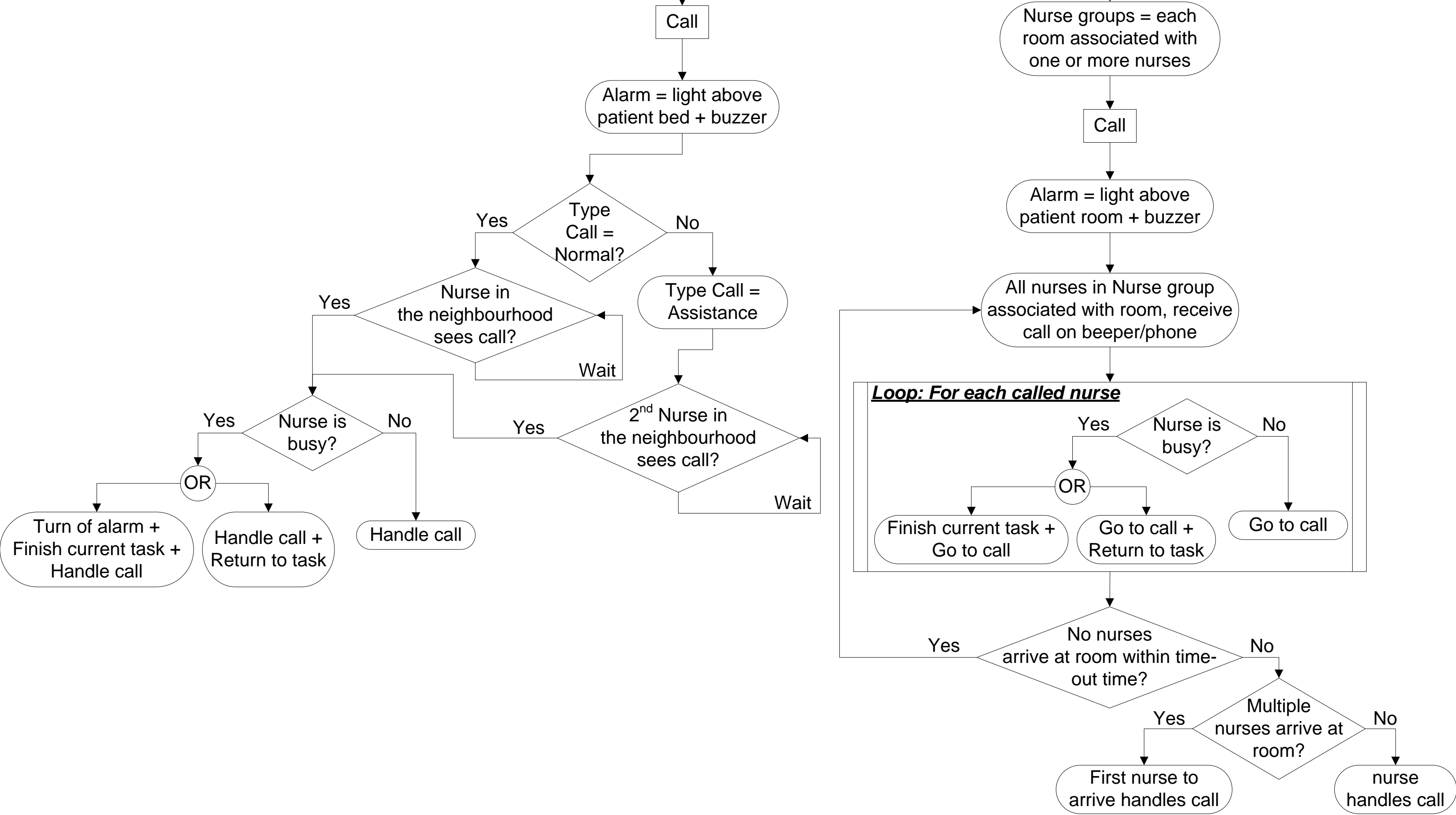


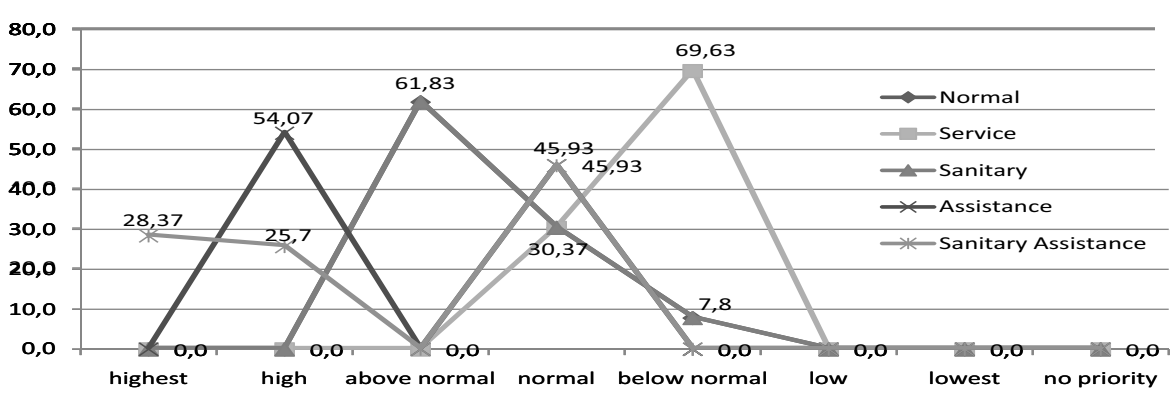

a) Kind of calls - test group

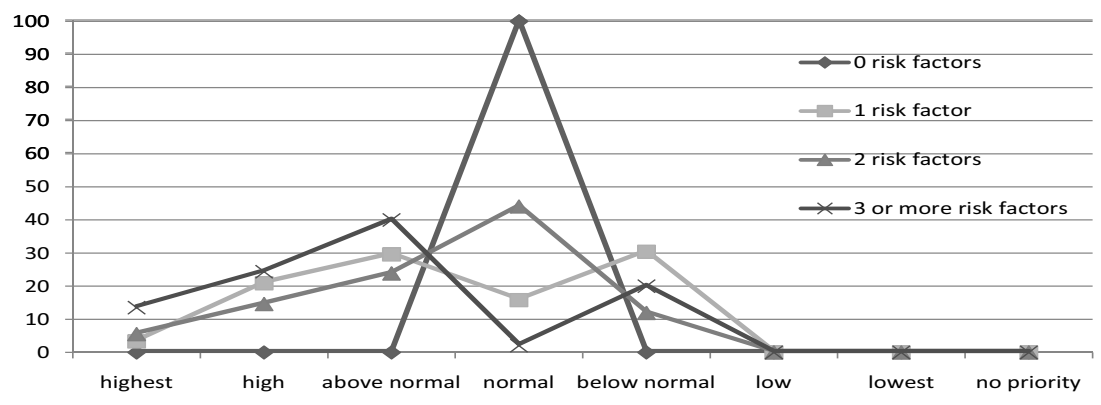

c) $\mathrm{Nr}$. of risk factors - test group

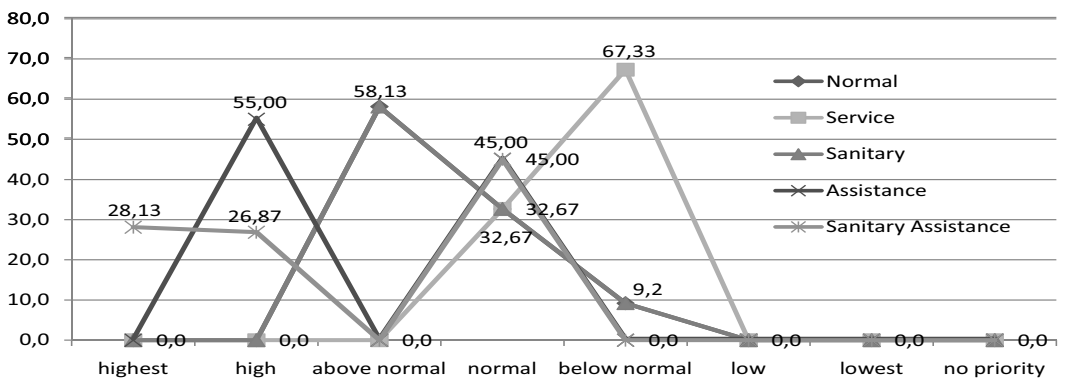

b) Kind of calls - validation group

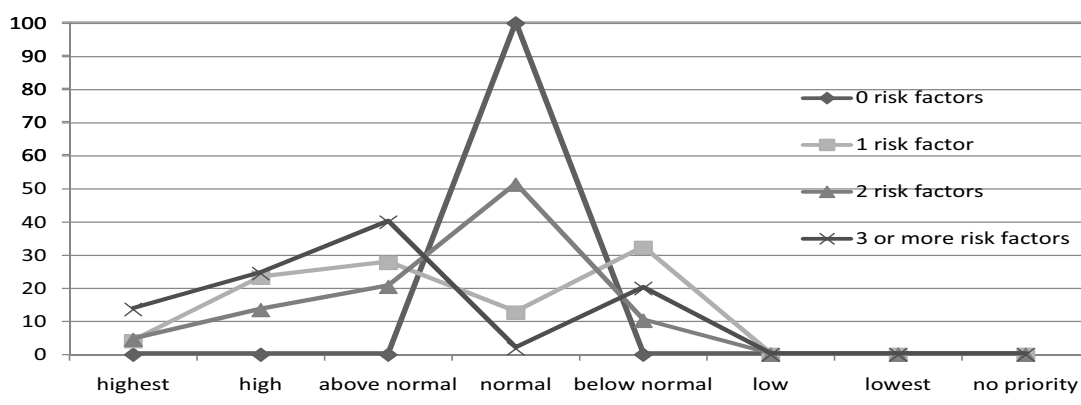

d) Nr. of risk factors - validation group 

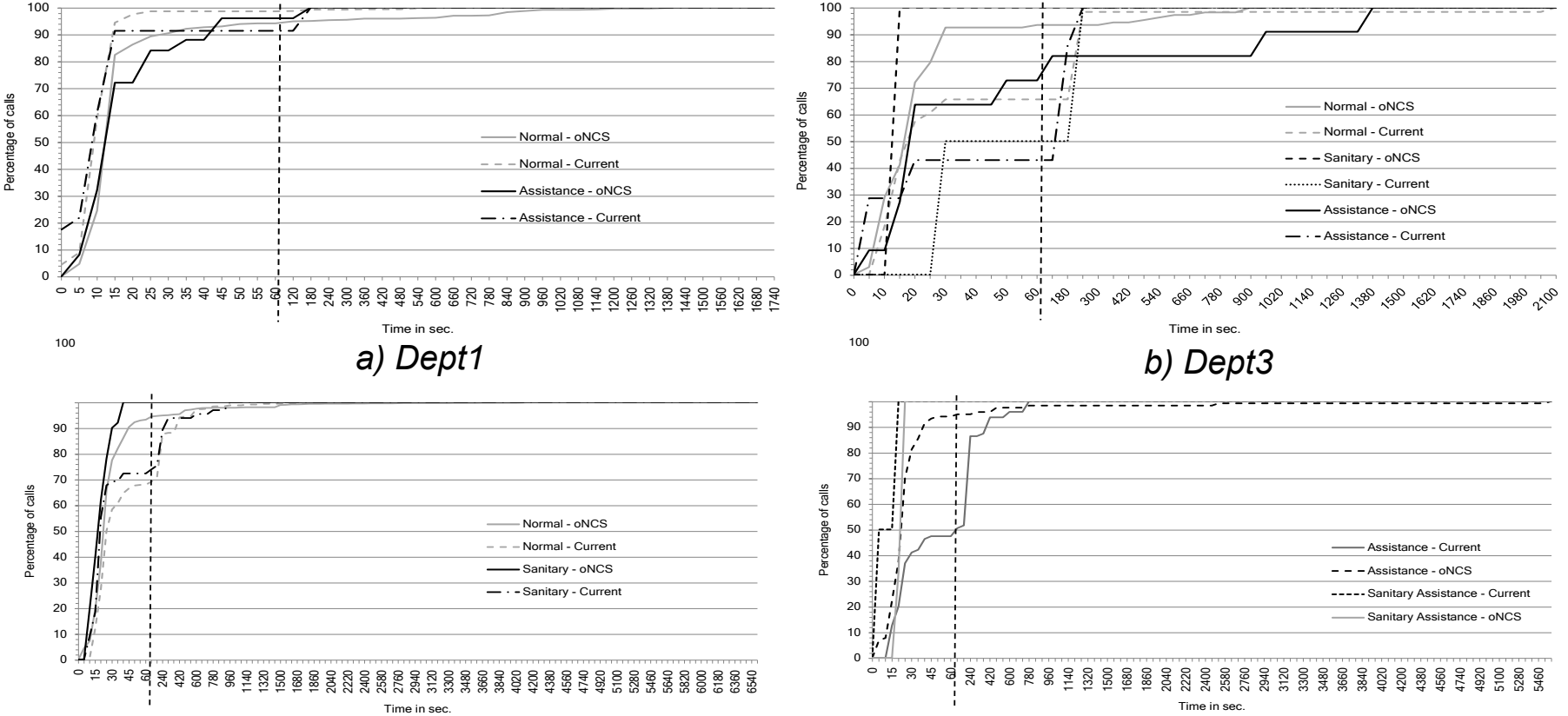

a) Dept2 - Normal, Sanitary calls

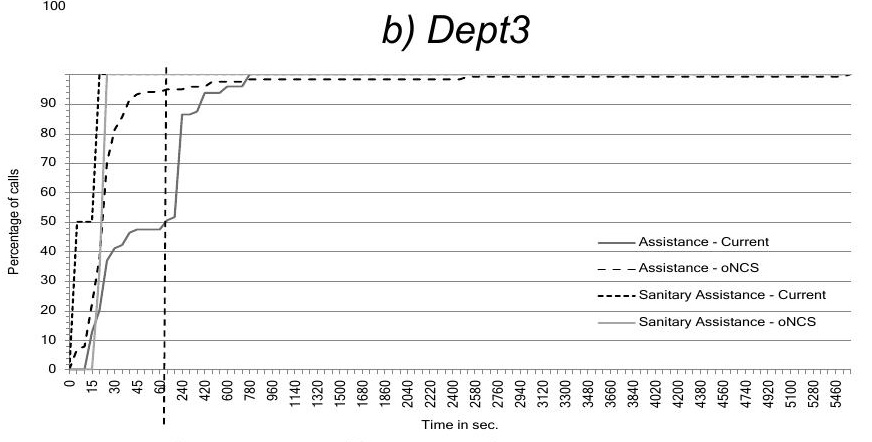
a) Dept2 - (Sanitary) Assistance calls 


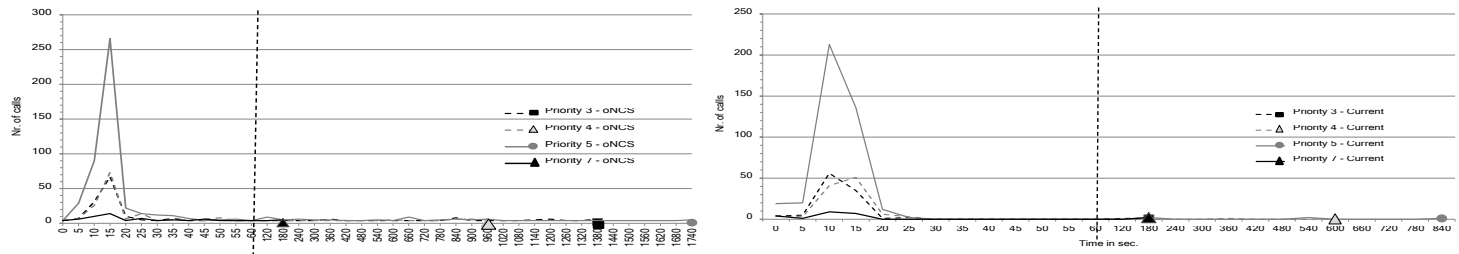

a) Dept1 - ONCS system

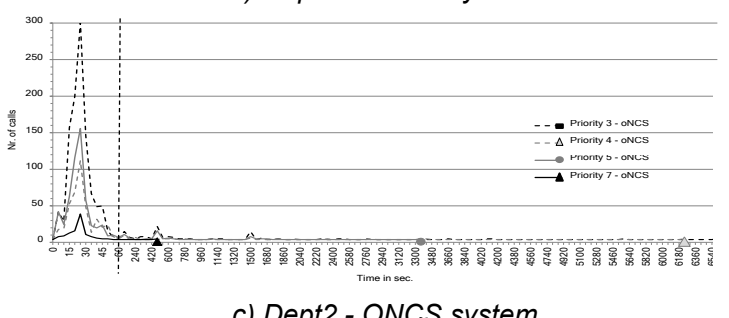

c) Dept2 - ONCS system

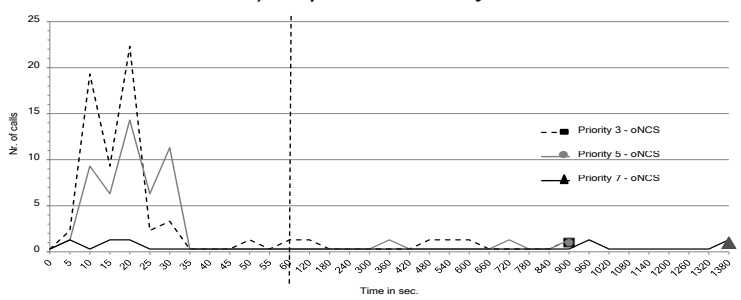

e) Dept3 - ONCS system b) Dept1 - Current system
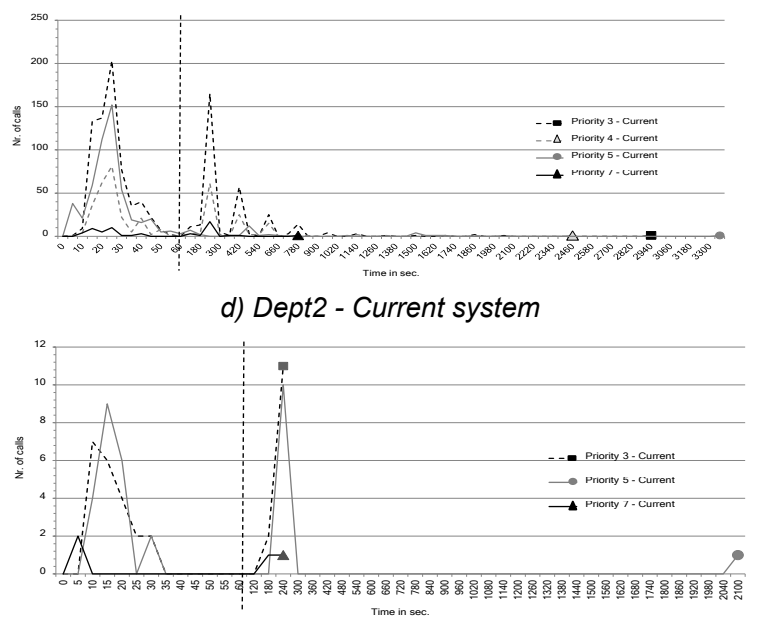

f) Dept3 - Current system 
